\title{
Correspondence
}

\section{Incident with a Baxter AS40A syringe pump: vigilance is warranted}

\section{To the Editor:}

Modern syringe pumps are designed to deliver medications in a safe manner. Alarm sensors incorporated into these devices should alert physicians when syringe loading is incomplete. A 40-yr-old lady was scheduled to undergo an elective vaginal hysterectomy. After spinal anesthesia was performed the Baxter AS40A pump was loaded with a $20 \mathrm{~mL}$ Becton Dickinson (BD) syringe containing propofol $(200 \mathrm{mg})$. The plunger clamp assembly was inadvertently not applied to the syringe. The infusion line was purged manually. The pump was then turned on and programmed to deliver $10 \mathrm{~mL} \cdot \mathrm{hr}^{-1}$ of propofol. The start button was depressed and the pump display confirmed the pump was in run mode (Figure). At no time did an alarm signal or error message appear on the display, to indicate that the plunger clamp was not applied to the syringe. The manufacturer's instruction booklet for this syringe pump states the following:

"Message: check syringe.

Cause: the syringe is improperly mounted.

Correction: make sure the syringe is centered in the barrel clamp cradle, and that the syringe flange (tab) is in the barrel clamp tab slot. Use the heel of the hand to press the barrel clamp firmly against the syringe".

The pump had last undergone a general service in June of 2003, and no problems had been documented at this time. The plunger clamp mechanism appeared to function normally on inspection and subsequent loading of the syringe. A repeat test of the entire events that occurred in setting up the pump, were performed after the case was complete.

The exact same findings as described above were documented. The medical manufacturer was notified of this incident. Baxter Canada indicated that this particular model does not have an alarm sensor for the plunger clamp. The Baxter AS50 pump does come with a sensor that would indicate "check plunger" in circumstances of incorrect plunger clamp loading. The Baxter AS40A syringe pump should also always be purged using the pump prior to commencement of infusions. The "purge operation" function advances the syringe plunger driver a preset distance. The primary purpose of this function is to eliminate slack in

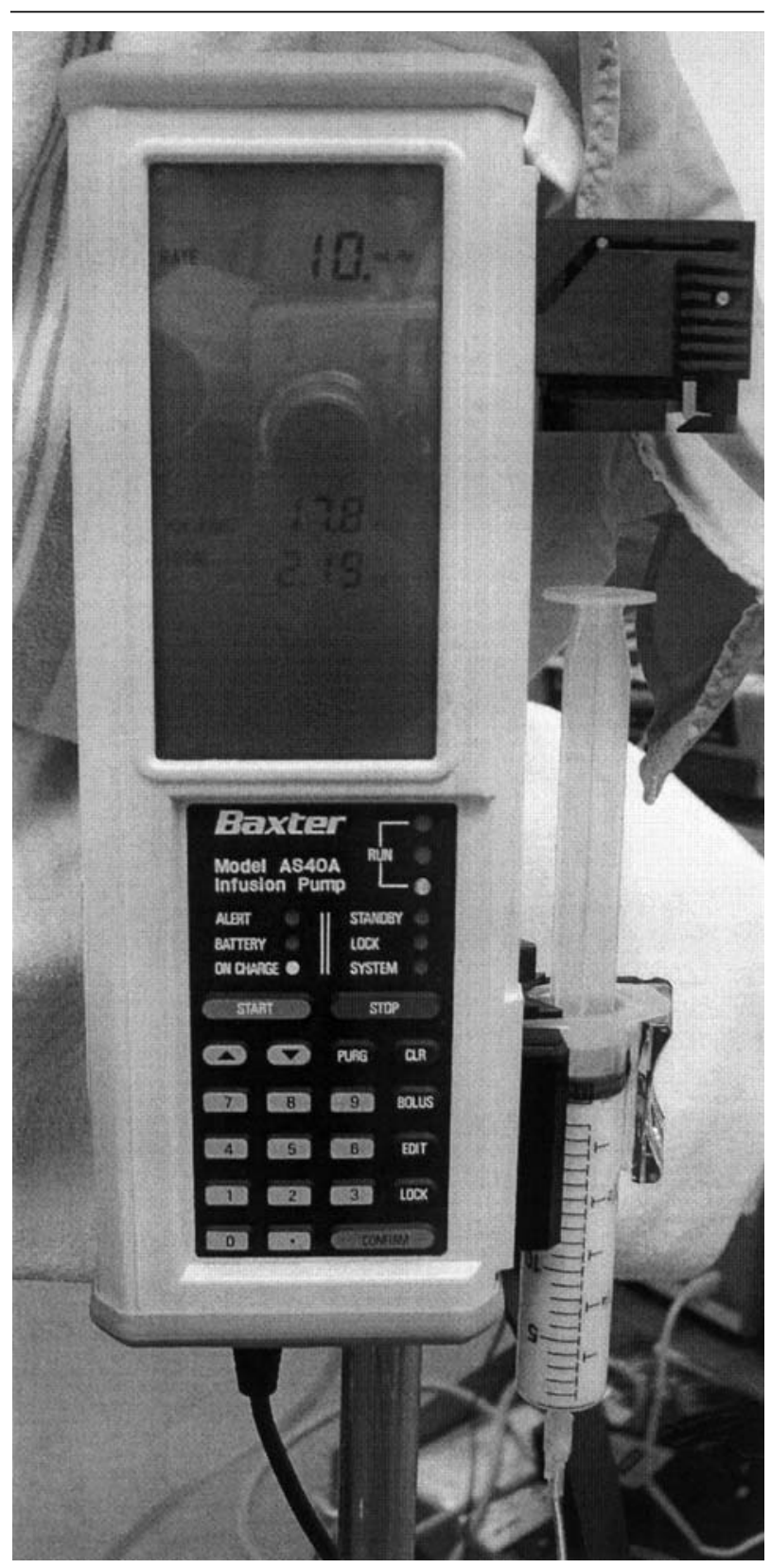

FIGURE Baxter AS40A pump in run mode without the plunger clamp assembly attached to the syringe. 
the drive mechanism, and thus ensure prompt fluid delivery when the infusion is started. Knowledge of the functioning of alarm systems of drug infusion devices, combined with astute vigilance is warranted to avoid potential adverse patient outcomes.

Chris Christodoulou MBCHB FRCPC

Winnipeg, Manitoba

Invited commentary:

The letter by Christodoulou illustrates a number of issues with equipment and its use.

Infusion pumps are one of the most potentially dangerous devices healthcare providers use on a regular basis. The FDA adverse event reports database $(\text { MAUDE })^{1}$ and Joint Commission on Accreditation of Health Care Organizations ${ }^{2}$ in the United States both document and warn of their dangers.

Despite this knowledge, very few persons read instruction manuals or understand all the features and limitations of their equipment. Alarm systems are not well understood, sometimes ignored and often disabled. $^{3}$

Equipment designers and manufacturers fail to adequately address and test the usability of equipment. The usability of a device includes how easy is it to learn, and how well one retains how to use a device over time, the efficiency of its use, whether the user likes to use the equipment and whether it is easy to correct errors when they have been made and recognized. ${ }^{4}$

The third point is the age old consumer advice "Caveat Emptor." Why was this pump in question purchased, when other pumps available on the market at the time of the Baxter AS40A's introduction to the market, had the syringe sensor available? The other question that is continuously asked is "why do we keep equipment in service that we know has problems?"

Manufacturers are often frustrated by the way people use their devices. Manufacturers are now required to mitigate risk of their equipment for reasonably foreseeable misuse, but often throw up their hands and state that they can't design for stupidity. Equipment is often used outside the manufacturer's intended environment of use, for purposes they have never foreseen.

As monies for capital equipment purchases are scarce and patient safety foremost, anesthesia departments must be vigilant, use due diligence when assessing and purchasing equipment and must have a formal evaluation procedure to ensure that problems like the one described by Christodoulou happen with less frequency.
Steven Dain MD FRCPC

London, Ontario

\section{References}

1 http://www.accessdata.fda.gov/scripts/cdrh/cfdocs /cfMAUDE/search.CFM.

2 http://www.jcaho.org/about+us/news+letters/sentinel+event+alert/sea_15.htm.

3 Vicente $K$. The Human Factor: Revolutionizing the Way People Live with Technology. Toronto: Random House Canada; 2003.

4 Nielsen J. Usability Engineering. San Francisco: Academic Press; 2003.

\section{Pressure alopecia in living donors for liver transplantation}

To the Editor:

We wish to report three living donor liver transplantation (LDLT) donors who developed occipital pressure alopecia (PA) in the perioperative period. All donors were healthy without past medical history. We had experienced about 100 LDLT surgeries until then. Anesthesia consisted of a combination of general and epidural anesthesia. General anesthesia was maintained with $\mathrm{O}_{2}-\mathrm{N}_{2} \mathrm{O}$-isoflurane, and paralysis with vecuronium. Throughout the operations each patient was placed in supine position while the hair was covered with a paper cap and the head was positioned on a soft foam doughnut made of urethane sponge covered with vinyl chloride. Surgeries were prolonged but uneventful. There were no episodes of intraoperative hypotension (systolic blood pressure $<90 \mathrm{mmHg}$ ), hypoxia $\left(\mathrm{SpO}_{2}<94 \%\right)$, or use of vasoconstrictor agents. All donors were extubated immediately after the operation.

The donors noticed occipital alopecia several days after the operation. Alopecia almost corresponded with the site of the circular headrest. All cases were treated with mometasone furoate ointment and received a scalp massage. We did not obtain skin biopsies and alopecia regressed in a few months.

Abel et al. reported eight gynecological patients who developed alopecia during the postoperative period and speculated that pressure-induced ischemia caused alopecia by resulting in the temporary cessation of follicle activity. ${ }^{1}$ Lawson et al. reported that localized scalp pressure was the cause of alopecia following cardiopulmonary bypass surgery, and the duration of pressure determined the extent of the damage. $^{2}$ The putative cause of PA is localizedinduced ischemia to the scalp caused by head immo- 